\title{
Valproic acid induces autophagy by suppressing the Akt/mTOR pathway in human prostate cancer cells
}

\author{
QINGHUA XIA $^{1}$, YI ZHENG ${ }^{2}$, WEI JIANG ${ }^{1}$, ZHONGXIAN HUANG $^{3}$, MUWEN WANG $^{1}$, \\ RONALD RODRIGUEZ ${ }^{3}$ and XUNBO JIN ${ }^{1}$ \\ ${ }^{1}$ Department of Minimally Invasive Urology, \\ Shandong Provincial Hospital Affiliated to Shandong University, Jinan, Shandong 250021; ${ }^{2}$ Department of Emergency, \\ Qilu Hospital of Shandong University, Jinan, Shandong 250012, P.R. China; ${ }^{3}$ Department of Urology, \\ University of Texas Health Science Center, San Antonio, TX 78229-3900, USA
}

Received February 11, 2015; Accepted May 10, 2016

DOI: $10.3892 / \mathrm{ol} .2016 .4880$

\begin{abstract}
Previous studies have demonstrated that the chronic administration of valproic acid (VPA) suppresses angiogenesis in vivo; however, the mechanisms implicated in VPA-induced autophagy remain unclear. The current study aimed to assess VPA-induced autophagy in three prostate cancer cell lines (PC3, DU145 and LNCaP), in addition to analyzing the Akt/mammalian target of rapamycin (mTOR) signal pathway. Prostate cancer cell lines were cultured with various doses of VPA. Cell cycle was analyzed using flow cytometry, and autophagy markers [1A/1B-light chain 3 (LC3)-II and Beclin-1] were examined using transmission electron microscopy, fluorescent microscopy and western blotting. Activation of the Akt/mTOR signal pathway was also assessed by western blotting. The results demonstrated that VPA induced autophagosomes and suppressed the Akt/mTOR signal pathway. This was confirmed by detection of increased LC3-II and Beclin-1 in VPA-treated cells compared with untreated controls. Phosphorylated forms of Akt (PC3, P=0.048; DU145, $\mathrm{P}=0.045$; LNCaP, $\mathrm{P}=0.039$ ) and mTOR (PC3, $\mathrm{P}=0.012$; DU145, $\mathrm{P}=0.41$; $\mathrm{LNCaP}, \mathrm{P}=0.35$ ) were significantly reduced following VPA treatment. These results suggest that VPA may function as a histone deacetylase inhibitor, suppressing the growth of prostate cancer cells by modulating autophagy pathways, including inhibition of the Akt/mTOR pathway. Further experiments are required to determine the significance of all involved pathways regarding VPA-induced growth inhibition.
\end{abstract}

Correspondence to: Dr Xunbo Jin, Department of Minimally Invasive Urology, Shandong Provincial Hospital Affiliated to Shandong University, 9677 Jingshidong Road, Jinan, Shandong 250021, P.R. China

E-mail: 328785635@qq.com

Key words: valproic acid, histone deacetylase inhibitor, prostate cancer, autophagy, Akt/mammalian target of rapamycin pathway

\section{Introduction}

In the USA, prostate cancer is the most prevalent malignancy among men and is the second most common cause of cancer-associated mortality (1). Although prostate cancer mortality rates declined steadily over recent years, it was estimated that there will be 180,890 new cases and 26,120 mortalities reported in 2016 , accounting for $\sim 21 \%$ of newly diagnosed male cancers in 2016 (2).

Histone acetylation/deacetylation is important in the post-translational modification of histones and is a vital mechanism in gene expression (3). Histone acetyltransferase and histone deacetylase (HDAC) are the key enzymes responsible for these reversible, post-translational modifications (4). It has been previously observed that several HDACs are aberrantly expressed or mutated in human disease, particularly in cancer; thus, these have been targeted therapeutically for the treatment of various forms of human cancer (5). HDAC inhibitors (HDACIs) are currently being assessed as anticancer drugs (6).

Valproic acid (VPA) is an established drug for the long-term treatment of seizure disorders, which has recently been classified among the HDACIs, resulting in an increasing interest in its application in cancer therapy (7). Previous studies have investigated the anti-tumor effect of VPA on prostate cancer, with results demonstrating that VPA inhibits the growth and angiogenesis of prostate cancer $(8,9)$, whilst a further study elucidated the induction of autophagy by VPA in prostate cancer (10). Autophagy has recently been discussed as a potential target in cancer, and Akt is a vital kinase implicated in the negative regulation of autophagy via mammalian target of rapamycin (mTOR) (11).

In the present study, the inhibitory effect of VPA on the $\mathrm{Akt} / \mathrm{mTOR}$ signaling pathway and its relevance to the induction of autophagy in prostate cancer was examined, providing a novel mechanism and therapeutic target for the treatment of prostate cancer, therefore, the current study aimed investigate these mechanisms further.

\section{Materials and methods}

Cell culture. Three human prostate cancer cell lines, PC3, DU145 and LNCaP, were obtained from the Chinese Academy 
of Sciences (Beijing, China) and Kunming Cell Bank of Chinese Academy of Sciences (Kunming, China). Cells were cultured at $37^{\circ} \mathrm{C}$, with an atmosphere of $95 \%$ air and $5 \% \mathrm{CO}_{2}$, in RPMI-1640 medium with L-glutamine (Thermo Fisher Scientific, Inc., Waltham, MA, USA) supplemented with $10 \%$ heat-inactivated fetal bovine serum (Thermo Fisher Scientific, Inc.) and $1 \%$ penicillin/streptomycin. Cells were cultured until they achieved $70-80 \%$ confluence and were harvested with $0.05 \%$ trypsin $/ 0.25 \mathrm{mmol} / 1$ ethylenediaminetetraacetic acid (Thermo Fisher Scientific, Inc.). VPA (1 mol/l; VPA sodium salt; Sigma-Aldrich, St. Louis, MO, USA) stock was prepared in RPMI-1640, and was filtered and sterilized through a $0.22-\mathrm{mm}$ filter.

Flow cytometry for cell cycle assay. PC3 and DU145 cell lines, which had been treated with 0.6, 1.2 and $2.5 \mathrm{mmol} / 1 \mathrm{VPA}$, were collected by trypsinization, washed with PBS 3 times, for 10 minutes each wash, and resuspended in cold PBS at $1 \times 10^{6}$ cells $/ \mathrm{ml}$. The cell suspension was added dropwise to an equal volume of cold $70 \%$ ethanol with continuous agitation. Resuspended cells were treated with PBS containing $0.1 \%$ Triton X-100 and $10 \mu \mathrm{g} / \mathrm{ml}$ RNase (pancreatic ribonuclease; Sigma-Aldrich) for $30 \mathrm{~min}$ at room temperature in the dark. Propidium iodide (PI; Sigma-Aldrich) was subsequently added to a final concentration of $50 \mu \mathrm{g} / \mathrm{ml}$. The samples were analyzed using an Accuri C6 Flow Cytometer (BD Biosciences, San Jose, CA, USA). The fluorescent emissions were collected through a $575 \mathrm{~nm}$ band-pass filter for PI. At least 10,000 cells/sec were analyzed using FlowJo 7.6 software (FlowJo LLC, Ashland, OR, USA). .

Transmission electron microscopy. The effect of VPA treatment on ultrastructures in PC3, DU145 and LNCaP cells was detected using transmission electron microscopy. Briefly, each cell type $\left(2 \times 10^{5}\right)$ was plated in a $100-\mathrm{mm}$ Petri dish, incubated overnight at $37^{\circ} \mathrm{C}$ and treated with VPA $(2.5 \mathrm{mmol} / \mathrm{l})$ for $48 \mathrm{~h}$ at $37^{\circ} \mathrm{C}$. Samples without VPA served as a control group. Cells were harvested, pelleted, fixed in $3 \%$ glutaraldehyde (Sigma-Aldrich) for $2 \mathrm{~h}$ at $4^{\circ} \mathrm{C}$ and finally postfixed with $1 \%$ osmium tetroxide (Sigma-Aldrich) at $37^{\circ} \mathrm{C}$ for $1 \mathrm{~h}$. The samples were then rinsed with water, dehydrated in a graded series of alcohol (50, 70 and 90\% alcohol) and kept in 90\% alcohol/90\% acetone (dilution, 1:1), followed by dehydration in a graded series of acetone (90-100\%). Subsequent to rinsing with acetone/Poly Bed (Polysciences, Warrington, PA, USA) at proportions of $3: 1,1: 1$ and 1:3, the samples were embedded in Poly Bed and cured at $37^{\circ} \mathrm{C}$ for $12 \mathrm{~h}, 45^{\circ} \mathrm{C}$ for $12 \mathrm{~h}$ and $60^{\circ} \mathrm{C}$ for $24 \mathrm{~h}$. Ultrathin sections $(50-70 \mathrm{~nm})$ were cut using a Leica RM2235 ultramicrotome (Leica Microsystems GmbH, Wetzlar, Germany) and observed with a transmission electron microscope (Hitachi H-800; Hitachi, Tokyo, Japan).

Fluorescent microscopy. Prostate cancer cell lines, DU145 and LNCaP, were grown in 6-well, flat-bottomed, microtiter plates $\left(5 \times 10^{5}\right.$ cells/well), incubated overnight and cultured in medium with VPA (2.5 mmol/l for $48 \mathrm{~h}$ ). Samples without VPA served as a control group. Cells were washed with PBS and fixed for 15 min using 4\% paraformaldehyde (Merck \& Co., Inc., Whitehouse Station, NJ, USA). Subsequently, the cells were permeabilized with $0.1 \%$ Triton X-100 (Sigma-Aldrich) for
$25 \mathrm{~min}$ at room temperature, washed twice with PBS for $10 \mathrm{~min}$ and blocked with PBS containing $0.5 \%(\mathrm{w} / \mathrm{v})$ bovine serum albumin (BSA; Biological Industries, Kibbutz Beit-Haemek, Israel) for $30 \mathrm{~min}$ at room temperature. Cells were treated with primary rabbit anti-human 1A/1B-light chain 3 (LC3)-II (catalog no., sc-134226; dilution, 1:1,000 in BSA; Santa Cruz Biotechnology, Inc., Dallas, TX, USA) and Beclin-1 antibodies (catalog no., sc-10086; dilution, 1:100 in BSA; Santa Cruz Biotechnology, Inc.) at $4^{\circ} \mathrm{C}$ overnight, washed with PBS 3 times and subsequently incubated with secondary goat anti-rabbit fluorescein isothiocyanate-conjugated antibody DyLight 549-conjugated antibody (catalog no., ab6721; dilution, 1:5,000 in BSA; Abcam, Cambridge, UK) for $30 \mathrm{~min}$ at $37^{\circ} \mathrm{C}$, avoiding light. The cells were then washed with PBS 3 times, dyed in 4',6-diamidino-2-phenylindole (Roche Diagnostics, Basel, Switzerland) for $30 \mathrm{~min}$ and finally observed under a fluorescent microscope (Olympus IX70; Olympus Corporation, Tokyo, Japan).

Western blot analysis. PC3, DU145, and LNCaP cell lines, which had been cultured to $70-80 \%$ confluence in $100-\mathrm{mm}$ Petri dishes, were treated with complete medium containing 0, 2.5 and $5.0 \mathrm{mmol} / 1 \mathrm{VPA}$ for $48 \mathrm{~h}$. Cells were harvested, washed in PBS 3 times for 10 min each and lysed in $100 \mu 1$ mammalian protein extraction reagent (Beyotime Institute of Biotechnology, Haimen, China). A Bicinchoninic Acid Protein assay kit (Beijing Solarbio Science \& Technology Co., Ltd., Beijing, China) was used to determine total protein concentration, and purified BSA was used to generate the standard curve. Concentrated proteins were separated by gel electrophoresis on a $15 \%$ Tris-hydrochloride $(\mathrm{HCl})$ polyacrylamide (separating gel) or 4-18\% gradient gel (electrophoresis gel), and transferred to a polyvinylidene difluoride membrane (EMD Millipore, Billerica, MA, USA). The membrane was blocked for $1 \mathrm{~h}$ in blocking buffer (containing $100 \mathrm{mmol} / \mathrm{l}$ Tris- $\mathrm{HCl}, 150 \mathrm{mmol} / \mathrm{l} \mathrm{NaCl}$ and $0.1 \%$ Tween 20) with 5\% nonfat dry milk, and incubated with rabbit anti-human antibody (catalog no., ab6759; dilution, 1:100 in BSA; Abcam) overnight, followed by incubation with anti-rabbit immunoglobulin $\mathrm{G}$ horseradish peroxidase-conjugated antibody (Santa Cruz Biotechnology, Inc.) for $1 \mathrm{~h}$ at room temperature. Immunoreactive bands were detected using the enhanced chemiluminescence plus western blotting detection system (EMD Millipore) according to the manufacturer's protocol. Polyclonal anti- $\beta$-actin antibody (Santa Cruz Biotechnology, Inc.) was used to detect $\beta$-actin in the same blots, which served as a loading control. ImageJ $1.48 \mathrm{v}$ (National Institutes of Health, Bethesda, MD, USA) was used to quantify the results of western blot analysis.

Statistical analysis. Each experiment was performed in triplicate and data are expressed as the mean \pm standard deviation. One-way analysis of variance with Scheffe post-hoc comparison test was used for comparison of dose-dependent treatment effects. $\mathrm{P}<0.05$ was considered to indicate a statistically significant difference. All statistical tests were performed using GraphPad Prism version 5.0 (GraphPad Software, Inc., La Jolla, CA, USA). The present study was approved by the Ethics Committee of Shandong Provincial Hospital Affiliated to Shandong University (Jinan, China). 
A

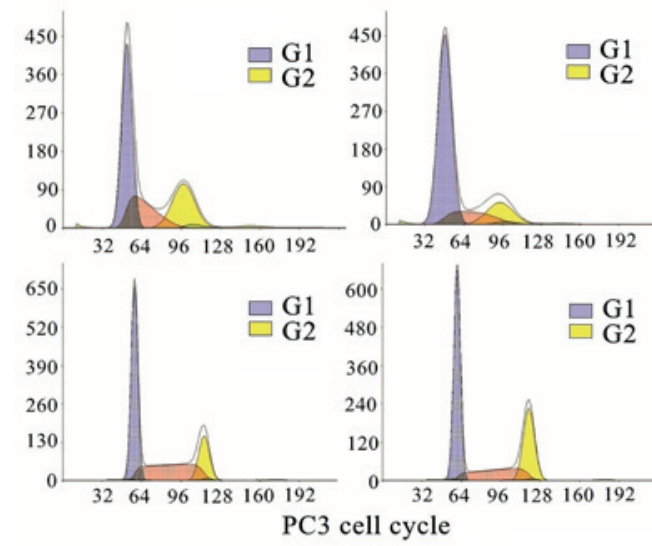

B

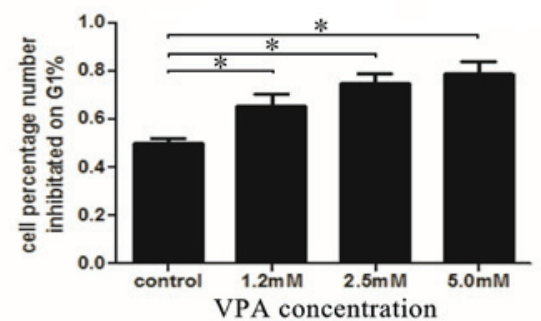

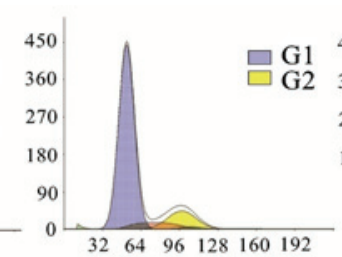
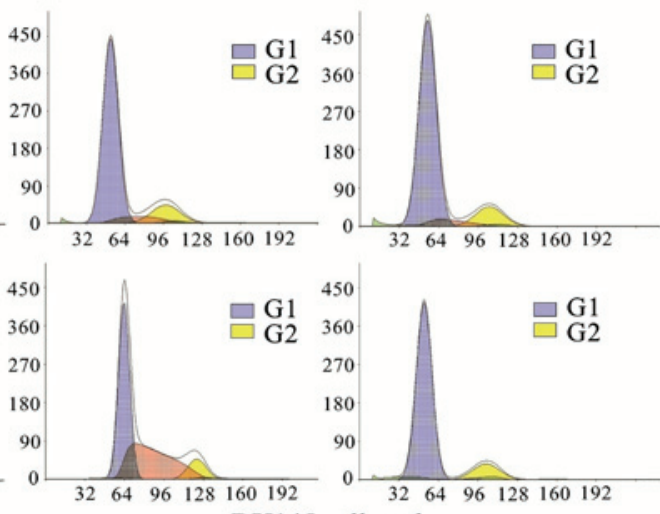

DU145 cell cycle

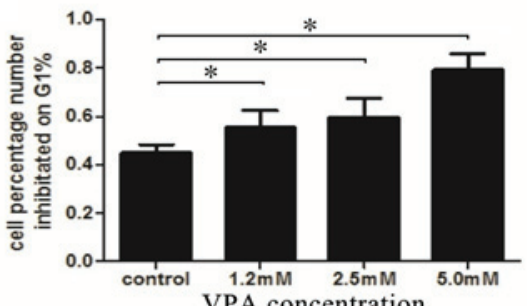

VPA concentration

Figure 1. VPA induces cell cycle arrest primarily at the $\mathrm{G}_{0}-\mathrm{G}_{1}$ phase. (A) Cells were treated with various concentrations of VPA (1.2,2.5 and 5.0 mmol/l) for $48 \mathrm{~h}$, and were subsequently fixed and stained with propidium iodide. Cell cycle distribution was assessed by flow cytometry. When the DNA curve was measured, the cell number formed a $G_{1}$ percentage peak. Subsequent to VPA treatment, the percentage of cells in the $G_{0}-G_{1}$ phase were significantly increased in a dose-dependent manner in PC3 and DU145 cells. VPA caused cell cycle arrest in PC3 and DU145 cell lines. (B) Histograms represent the percentage of cells arrested at the $G_{0}-G_{1}$ phase. The percentage of cells in the $G_{0}-G_{1}$ phase were increased in a dose-dependent manner in PC 3 and DU145 cells. The $G_{0}-G_{1}$ phase of the three VPA treatment groups was significantly longer compared with the control groups in the two cell lines. "P<0.05. VPA, valproic acid.
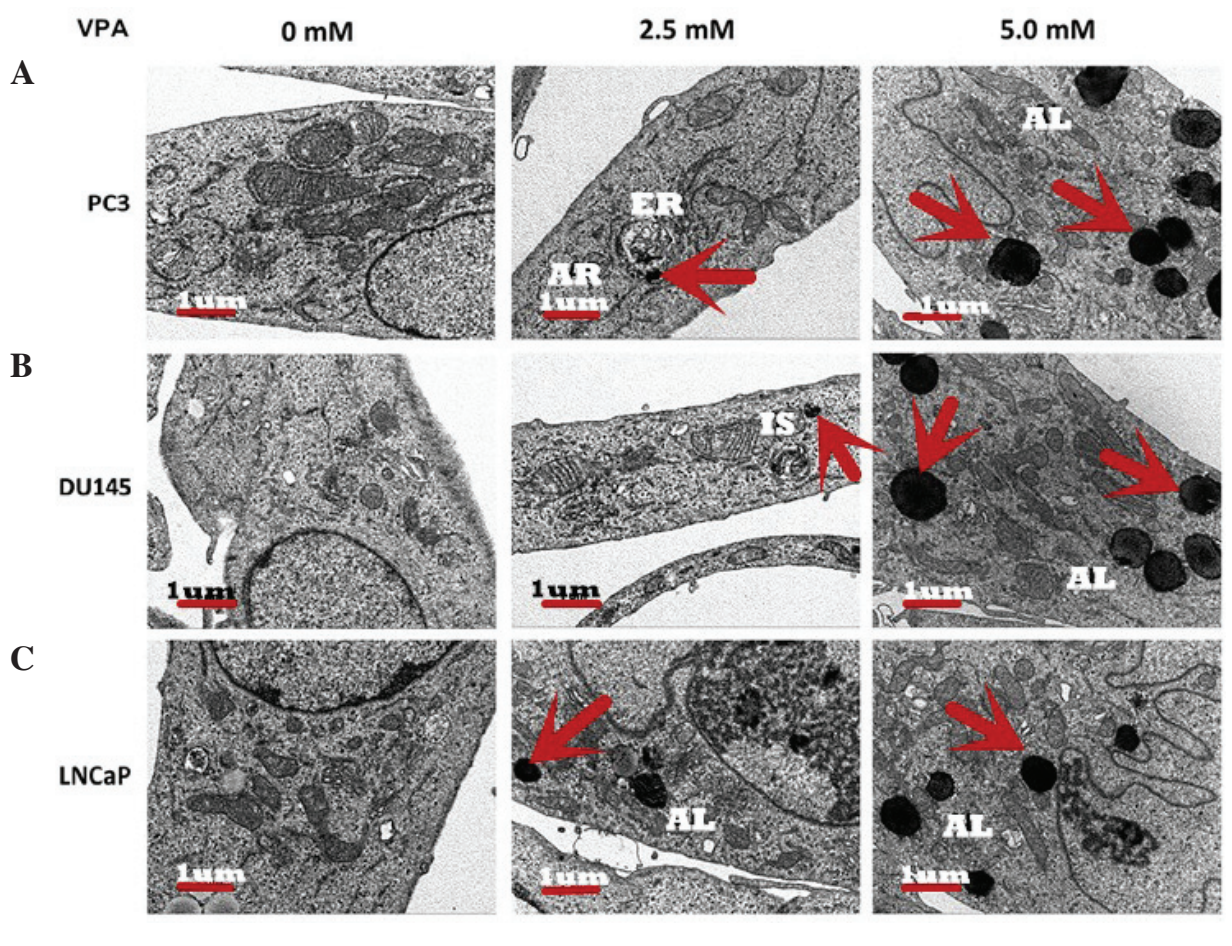

Figure 2. Ultrastructure of autophagy observed by electron microscopy in prostate cancer cells following treatment with valproic acid (0,2.5 and $5.0 \mathrm{mM})$. Different sizes and stages of autophagic vacuoles were visible at a x1,000 magnification. Numerous autophagic lysosomes (arrowheads) were observed in the (A) PC3, (B) DU145 and (C) LNCaP cells. The majority of autophagic lysosomes were identified in the late autophagic stages.

\section{Results}

VPA induces cell cycle arrest primarily at the $G_{0^{-}} G_{l}$ phase. To further understand the mechanism underlying VPA-induced cell growth inhibition, the impact of VPA on the regulation of cell cycle distribution was investigated using flow cytometry. As presented in Fig. 1, the results demonstrated that VPA was able to induce cell cycle arrest in the DU145 and LNCaP cell 
A

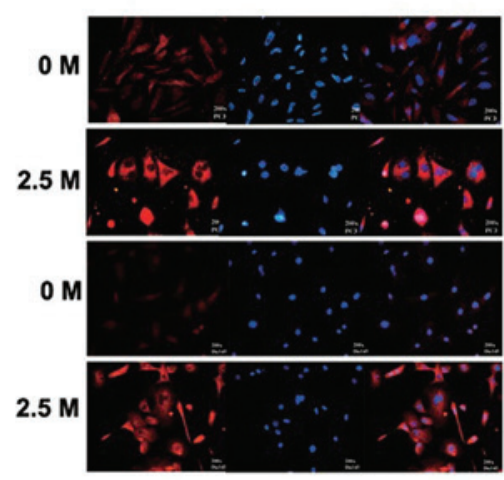

B

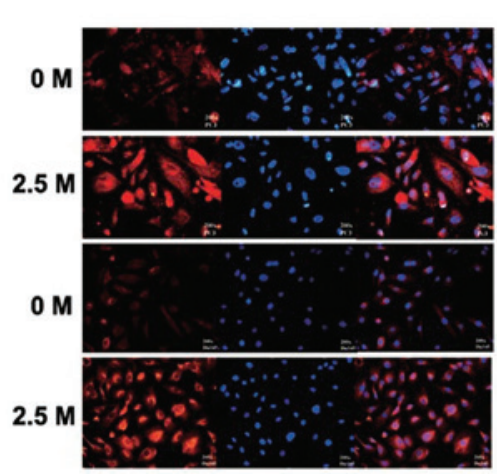

C

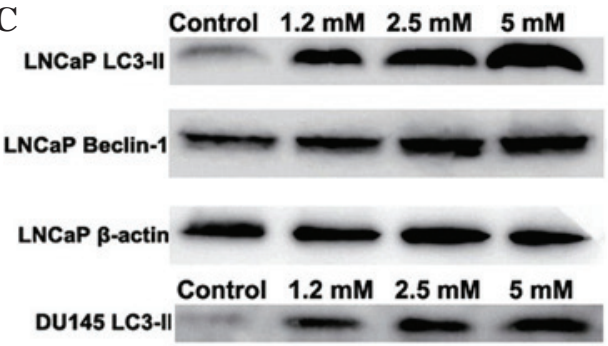

DU145 Beclin-1

DU145 $\beta$-actin

DU145 LC3॥

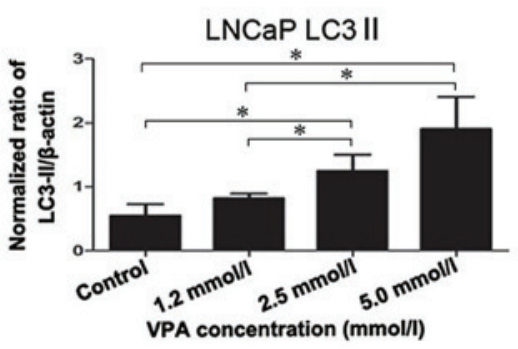

LNCaP Beclin-1

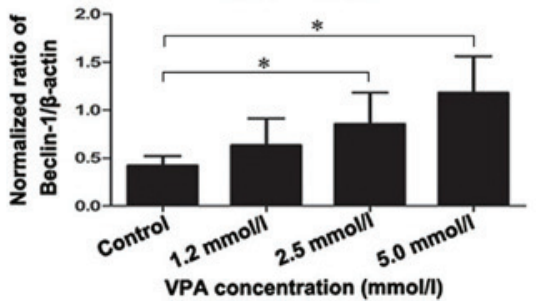

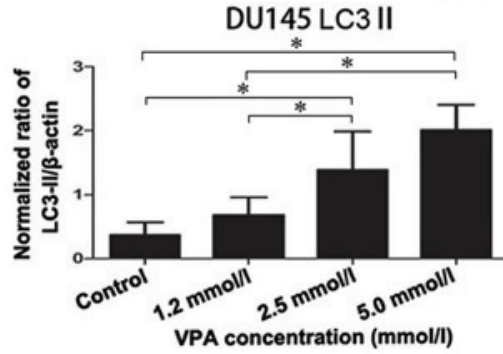

DU145 Beclin-1

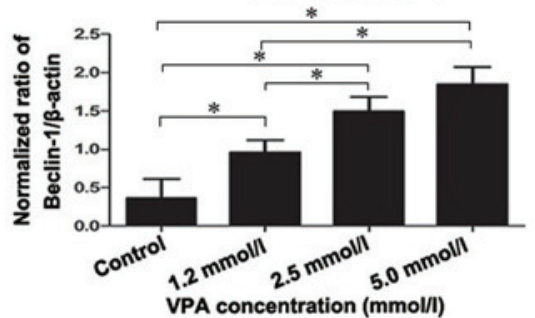

Figure 3. Expression of specific autophagic proteins, LC3-II and Beclin-1, in prostate cancer cells. There was an overlap in the distribution and expression of (A) LC3 and (B) Beclin-1 protein, which merged with DAPI nuclear staining (blue). Cells untreated or treated with VPA (2.5 mmol/1 for 48 h) were fixed and probed with polyclonal rabbit anti-LC3-II and anti-Beclin-1 antibodies, in addition to DyLight 549-conjugated (red) secondary antibodies. (C) Western blot analysis detected the induction of LC3-II and Beclin-1 protein expression in cells treated with VPA (1.2, 2.5 and 5.0 mmol/1) compared with the non-treated control. LC3-II and Beclin-1 expression increased in a dose-dependent manner in all cell lines after $48 \mathrm{~h}$ incubation with VPA. (D) Histograms representing the level of LC3-II and Beclin-1 expression in LNCaP and DU145 cells (normalized to $\beta$-actin). In LNCaP cells, LC3-II and Beclin-1 protein levels in the $2.5 \mathrm{mmol} / 1$ and $5.0 \mathrm{mmol} / 1 \mathrm{VPA}$ treatment groups were significantly increased compared with the control groups. In addition, $1.2 \mathrm{mmol} / 1 \mathrm{VPA}$ treatment did not significantly increase LC3-II and Beclin-1 protein levels. In DU145 cells, LC3-II protein levels in $2.5 \mathrm{mmol} / 1$ and 5.0 mmol/1 VPA treatment groups were significantly increased compared with the control groups. Beclin-1 protein levels in all three VPA treatment groups were significantly increased compared with the control groups. ${ }^{*} \mathrm{P}<0.05$. LC3, 1A/1B-light chain 3; VPA, valproic acid.

lines. The cell number percentage in the $G_{0}-G_{1}$ phase significantly increased in a dose-dependent manner compared with control groups (PC3, $\mathrm{P}=0.035$; DU145, $\mathrm{P}=0.037)$.

Ultrastructure of autophagy in prostate cancer cells. Scanning electron micrographs illustrated that following treatment with VPA, autophagosomes formed inside the cytoplasm of the prostate cancer cells and appeared to contain cellular organelles (Fig. 2). Micrographs demonstrated that the control group exhibited normal organelle morphology. Autophagic vesicles and autolysosomes were observed in organelles, including the mitochondria, endoplasmic reticulum and cytoplasmic materials, in the cells treated with VPA. At the initiation of cell autophagy in all three cell lines, numerous smooth endoplasmic reticulum-like isolation membranes were observed, which formed portions of the cytosol. These double-membrane structures allow the fusion of autophagosomes with lysosomes, resulting in the formation of autophagic lysosomes (12). Finally, the majority of mitochondria and other organelles had disappeared from the cells, and remnants of organelles were identified in the autophagic lysosomes of VPA-treated cells whose nuclear chromatin had condensed into small, irregular masses.

Expression of specific autophagic proteins, LC3-II and Beclin-1, in DU145 and LNCaP cells. Accumulation of LC3-II is associated with the extent of autophagosome formation (13). Following DyLight-549 staining, the cells in the VPA treatment group exhibited red fluorescence in the cytoplasm, but not in the nucleolus, and the fluorescence was stronger than that of the non-treated control group, thus indicating the formation of autophagolysosomes (Fig. 3A). These autophagic changes were further confirmed by western blotting as presented in Fig. 3C and D (LNCaP, P=0.019; DU145, P=0.043). Furthermore, Beclin-1 and LC3-II exhibited strong immunofluorescence in the cytoplasm of the DU145 and LNCaP cells compared with the non-treated control group (Fig. 3A and B), which was also detected by western blot analysis ( $\mathrm{LNCaP}$, $\mathrm{P}=0.033$; DU145, $\mathrm{P}=0.027$ ) (Fig. 3C and3D). 

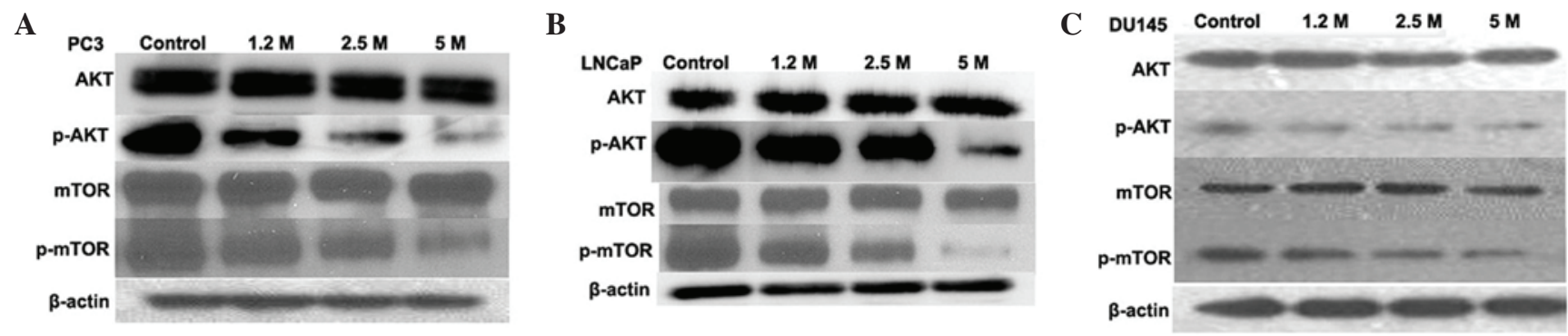

D
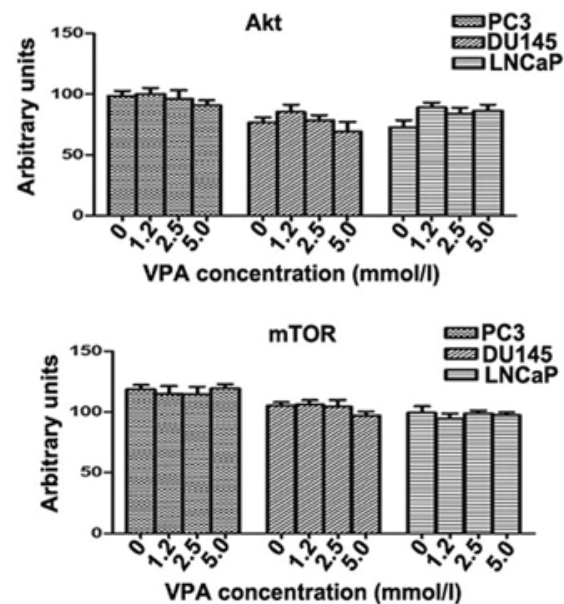
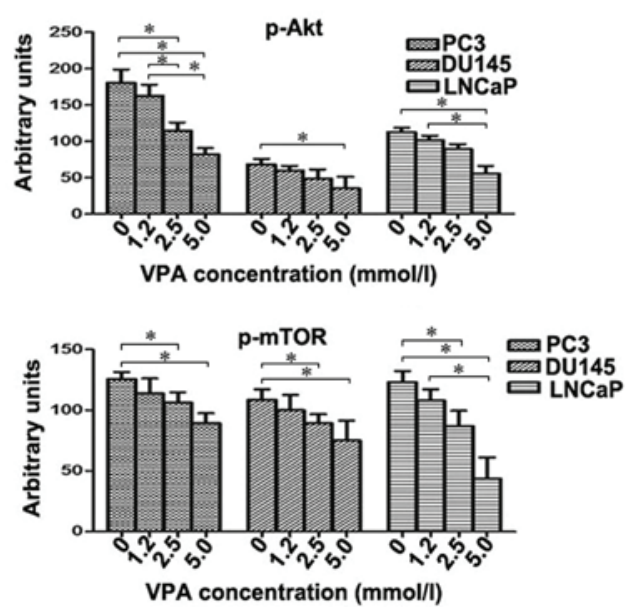

Figure 4. Effect of VPA on Akt, mTOR and their phosphorylated forms. Western blotting measured the levels of Akt, mTOR, and their phosphorylated forms in (A) PC3, (B) LNCaP and (C) DU145 cells subsequent to VPA treatment at various concentrations. (D) In contrast to the control group, VPA-treated cells were found to demonstrate a dose-dependent decrease in the phosphorylation of Akt and mTOR proteins in DU145, PC3 and LNCaP cells. There was no significant change in the protein levels of total Akt and mTOR. In addition, $5.0 \mathrm{mmol} / \mathrm{l}$ VPA treatment induced statistically significant decreases in p-mTOR protein levels in all three cell lines; $2.5 \mathrm{mmol} / 1$ and $5.0 \mathrm{mmol} / 1 \mathrm{VPA}$ treatment induced statistically significant decreases in p-mTOR protein levels in all three cell lines. *P<0.05. p-, phosphorylated; mTOR, mammalian target of rapamycin; VPA, valproic acid.

VPA inhibits activation of the Akt/mTOR pathway in prostate cancer cell lines. The Akt/mTOR signaling pathway was investigated in PC3, DU145 and LNCaP cell lines treated with VPA. The relative fold of phosphorylated Akt and mTOR increased as determined by scanning densitometry of the western blot normalized to $\beta$-actin. VPA-treated cells were observed to induce a dose-dependent decrease in the phosphorylation of Akt (PC3, $\mathrm{P}=0.048$; DU145, $\mathrm{P}=0.045$; LNCaP, $\mathrm{P}=0.039$ ) and mTOR (PC3, $\mathrm{P}=0.012$; DU145, $\mathrm{P}=0.41$; LNCaP, $\mathrm{P}=0.35$ ) protein compared to the control group (Fig. 4) in all cell lines. However, the total protein levels of Akt (PC3, $\mathrm{P}=0.082$; $\mathrm{DU} 145, \mathrm{P}=0.065$; LNCaP, $\mathrm{P}=0.059)$ and mTOR $(\mathrm{PC} 3, \mathrm{P}=0.12$; DU145, $\mathrm{P}=0.095$; LNCaP, $\mathrm{P}=0.089)$ did not change following VPA treatment $(\mathrm{P}>0.05)$ (Fig. 4D), which indicates that VPA inflicts a potent inhibitory effect on Akt/mTOR signaling.

\section{Discussion}

The two forms of programmed cell death, autophagic cell death and apoptosis, are not mutually exclusive and may contribute to oncogenesis and mortality (14). Traditionally, autophagic cell death is characterized by the formation of autophagic vacuoles, and regulation of the caspase family performs diversified roles in pathophysiology and physiology in various cell types $(11,15)$. In the tumor microenvironment, metabolic stress, including hypoxia and ischemia, may induce autophagy, which provokes cancer cells to autodigest and recycle damaged organelles by balancing its intracellular homeostasis to survive (16). By contrast, autophagy may also be involved in the degeneration of cell structures, inducing cell death subsequent to the activation of death signals. In addition, it was previously determined that there may be an association between tumorigenesis and the absence of autophagy genes, including Beclin-1, autophagy related (ATG) 5 and ATG7 (17).

HDACIs have previously undergone testing against oncogenesis due to their potential ability to reverse epigenetic changes associated with cancer cells (18). It has been reported that HDACIs are able to induce tumor cell death with similar physiological and morphological characteristics to those of apoptosis, particularly as they are selective and exert minimal toxic effects on the host (19). In a previous study, it was demonstrated that VPA significantly decreased the proliferation of prostate cancer cells in vitro and reduced tumor volume (20). It was considered that this antitumor activity may be associated with temporary cell arrest and downregulated expression of androgen receptors induced by chronic VPA treatment (11). The autophagy phenomenon has also been observed following a short period ( $<4$ days) of VPA administration in PC3 cells (10). In the present study, VPA was identified to inhibit prostate cancer cell growth in a dose-dependent manner, affirming the antineoplastic effect of VPA. Flow cytometry analysis demonstrated that the cell number percentage in the $\mathrm{G}_{1}$ phase significantly increased in the higher dose drug group, but the cell number in the $\mathrm{G}_{2} \%$ peak did not show an increase or decrease with increasing drug doses. The induction of cell cycle arrest by VPA highlights its antineoplastic role. This 
phenomenon may be explained by the knowledge that HDACIs activate cell death, resulting in cell cycle arrest in $\mathrm{G}_{1}-\mathrm{G}_{2}$. The $\mathrm{G}_{1}$ arrest induced by VPA in the present study was in accordance with the reported effect of this drug in endometrial carcinoma cell lines (15).

Chronic VPA treatment is expected to produce a more profound effect on cancer cell proliferation compared with standard VPA treatment, which may have limited activity. The cyclin-dependent kinase inhibitor, p21WAF/CIP1, is consistently induced by VPA and is key for the inhibition of cell growth (21). Cell death is typically associated with apoptosis; however, it may also occur through alternative mechanisms, including non-lysosomal vesiculate cell death and autophagy (22). The phenomenon of autophagy in response to antitumor therapies may be monitored by immunohistochemical analysis employing anti-lysosome-associated membrane protein 1 and anti-LC3B antibodies (23). Previously, it was reported that VPA was able to initiate a moderate apoptotic response through preferential activation of the mitochondrial pathway in prostate cancer cell lines (24). The results of the present study demonstrated that VPA may also induce prostate cancer cell death through the autophagy pathway. The presence of autophagic vacuoles in cancer cells following VPA treatment indicated that they were undergoing autophagy-related cell death (Fig. 2). Electron microscopy identified a number of large vacuoles in the cytoplasm in VPA treated groups, which were seldom observed in the control group (Fig. 2). These vacuoles exhibited typical morphological features of autophagy with a double ' $\mathrm{C}$ ' formation at the membrane origin of autophagosomes. It is generally considered that the mitochondria, plasma membrane or Golgi bodies may function as the primary membrane source for autophagosomes and other related structures (25). The present study observed that the initial autophagic ultrastructures emerged around these organelles in the VPA treatment group (Fig. 2), and the bulk of the cytoplasm and certain organelles were observed to be wrapped into the vacuole, and the autophagosome had merged with the lysosome.

Autophagosomes appear in the cytoplasm at the first stage of autophagy-associated cell death, and microtubule-associated LC3, particularly LC3-II, serves as an autophagosome-specific protein (26). LC3 is one of the most credible markers of autophagosomes in mammalian cells (27). LC3-I is cytoplasmic, whilst LC3-II is a tight membrane-bound protein that attaches to autophagosomes, which subsequently fuse with lysosomes (28). Relative amounts of membrane-bound LC3-II reflects the abundance of autophagosomes with the process that transforms LC3-I into LC3-II; thus, the induction and inhibition of autophagy is able to be monitored by immunoassay through the measurement of LC3-II levels (29). It has been reported that autophagy is suppressed in various types of cancer cells, and that cellular autophagic activity is inversely correlated with malignancy (30). Beclin-1 may also function as a marker of autophagy, which has been expressed in a monoallelic manner in human prostate, ovarian and breast cancer, which suggested that the process of autophagy may possess tumor-suppressor properties $(31,32)$. In the current study, western blot analysis demonstrated that LC3-II and Beclin-1 expression increased with VPA in a dose-dependent manner in prostate cancer cells, which was also observed by fluorescence microscopy (Fig. 3).

Various signaling pathways, such as autophagy-related (Atg) proteins, ULK and the Bcl-2 family, were involved in this process, were involved in this process, which consist of the core autophagic delivery to cell death (33). In yeast and mammalian cells, the Ras and mTOR pathways are two well-known signaling cascades that are sensitive to nutrient status, cell growth and differentiation, and are negatively regulated during programmed cell death (34). The phosphoinositide 3-kinase/Akt/mTOR pathway exists in several types of cancer and may be activated by the loss of tumor suppressor phosphatase and tensin homolog (PTEN) function (35). The formation of an autophagosome membrane may be affected by regulating the recruitment of the transmembrane protein ATG9, which facilitates lipid assembly to expand autophagosomes (36). This step is regulated by mTOR kinase, but the intracellular mechanism is remains unclear. The activation of Akt and its phosphorylation induces the expression of p21WAF and p27Kip1, which are associated with cell cycle growth through the acetylation of relevant genes (37). The present study demonstrated that treatment with VPA inhibited the activity of Akt and mTOR, resulting in a depletion of phosphorylated (p)-AKT and p-mTOR, which is considered to occur due to VPA concomitantly inducing p27 and p21. This may subsequently result in cell cycle arrest, growth inhibition and PTEN-loss-induced activation of the Akt pathway in prostate cancer cells. It is suspected that VPA-induced autophagic cell death may be involved in this process.

In conclusion, the present study provided evidence that VPA may function as an HDACI to suppress the growth of prostate cancer cells through the modulation of autophagic pathways, including inhibition of the Akt/mTOR pathway. Further experiments are required to determine the significance of each pathway involved in VPA-induced growth inhibition.

\section{Acknowledgements}

The present study was sponsored by the Department of Science and Technology of Shandong Province (grant no. BS2010YY047).

\section{References}

1. Antonarakis ES, Armstrong AJ, Dehm SM and Luo J: Androgen receptor variant-driven prostate cancer: Clinical implications and therapeutic targeting. Prostate Cancer Prostatic Dis: May 17, 2016 (Epub ahead of print).

2. Siegel RL, Miller KD and Jemal A: Cancer statistics, 2016. CA Cancer J Clin 66: 7-30. 2016.

3. Jiang W, Zheng Y, Huang Z, Wang M, Zhang Y, Wang Z, Jin X and Xia Q: Role of SMAD4 in the mechanism of valproic acid's inhibitory effect on prostate cancer cell invasiveness. Int Urol Nephrol 46: 941-946, 2014.

4. Hegde M, Mantelingu K, Pandey M, Pavankumar CS Rangappa KS and Raghavan SC: Combinatorial study of a novel poly (ADP-ribose) polymerase inhibitor and an HDAC inhibitor, SAHA, in leukemic cell lines. Target Oncol: May 17, 2016 (Epub ahead of print).

5. Barneda-Zahonero B and Parra M: Histone deacetylases and cancer. Mol Oncol 6: 579-589, 2012.

6. Giannini G, Cabri W, Fattorusso C and Rodriquez M: Histone deacetylase inhibitors in the treatment of cancer: Overview and perspectives. Future Med Chem 4: 1439-1460, 2012. 
7. Chateauvieux S, Morceau F, Dicato $M$ and Diederich $M$ Molecular and therapeutic potential and toxicity of valproic acid. J Biomed Biotechnol 2010: 479364, 2010.

8. Xia Q, Sung J, Chowdhury W, Chen CL, Höti N, Shabbeer S, Carducci M and Rodriguez R: Chronic administration of valproic acid inhibits prostate cancer cell growth in vitro and in vivo. Cancer Res 66: 7237-7244, 2006.

9. Gao D, Xia Q, Lv J and Zhang H: Chronic administration of valproic acid inhibits PC3 cell growth by suppressing tumor angiogenesis in vivo. Int J Urol 14: 838-845, 2007.

10. Huang ZX, Jin XB, Wang MW, Zhang YN, Zheng Y and Xia QH Effect of valproic acid on autophagy in prostate cancer PC 3 cells. Shandong Da Xue Xue Bao 49: 44-47, 2011 (In Chinese).

11. Nho RS and Hergert P: IPF fibroblasts are desensitized to type I collagen matrix-induced cell death by suppressing low autophagy via aberrant Akt/mTOR kinases. PLoS One 9: e94616, 2014.

12. Lauritzen I, Pardossi-Piquard R, Bourgeois A, Pagnotta S Biferi MG, Barkats M, Lacor P, Klein W, Bauer C and Checler F: Intraneuronal aggregation of the $\beta$-CTF fragment of APP (C99) induces $\mathrm{A} \beta$-independent lysosomal-autophagic pathology. Acta Neuropathol: Apr 30, 2016 (Epub ahead of print).

13. Goode A, Butler K, Long J, Cavey J, Scott D, Shaw B, Sollenberger J, Gell C, Johansen T, Oldham NJ, et al: Defective recognition of LC3B by mutant SQSTM1/p62 implicates impairment of autophagy as a pathogenic mechanism in ALS-FTLD. Autophagy: May 9, 2016 (Epub ahead of print).

14. Castagna C, Merighi A and Lossi L: Cell death and neurodegeneration in the postnatal development of cerebellar vermis in normal and Reeler mice. Ann Anat: Feb 28, 2016 (Epub ahead of print).

15. Zhao X, Yang W, Shi C, Ma W, Liu J, Wang Y and Jiang G: The G1 phase arrest and apoptosis by intrinsic pathway induced by valproic acid inhibit proliferation of BGC-823 gastric carcinoma cells. Tumour Biol 32: 335-346, 2011.

16. Kouidhi S, Noman MZ, Kieda C, Elgaaied AB and Chouaib S: Intrinsic and tumor microenvironment-induced metabolism adaptations of $\mathrm{T}$ cells and impact on their differentiation and function. Front Immunol 7: 114, 2016.

17. Chen Y, Liu XR, Yin YQ, Lee CJ, Wang FT, Liu HQ, Wu XT and Liu J: Unravelling the multifaceted roles of Atg proteins to improve cancer therapy. Cell Prolif 47: 105-112, 2014.

18. Damaskos C, Karatzas T, Nikolidakis L, Kostakis ID, Karamaroudis S, Boutsikos G, Damaskou Z, Kostakis A and Kouraklis G: Histone deacetylase (HDAC) inhibitors: Current evidence for therapeutic activities in pancreatic cancer. Anticancer Res 35: 3129-3135, 2015.

19. Carafa V, Miceli M, Altucci L and Nebbioso A: Histone deacetylase inhibitors: A patent review (2009-2011). Expert Opin Ther Pat 23: 1-17, 2013

20. Lee JE and Kim JH: Valproic acid inhibits the invasion of PC3 prostate cancer cells by upregulating the metastasis suppressor protein NDRG1. Genet Mol Biol 38: 527-533, 2015.

21. Nagai H, Fujioka-Kobayashi M, Ohe G, Hara K, Takamaru N, Uchida D, Tamatani T, Fujisawa K and Miyamoto Y: Antitumour effect of valproic acid against salivary gland cancer in vitro and in vivo. Oncol Rep 31: 1453-1458, 2014.
22. Cerella C, Teiten MH, Radogna F, Dicato $M$ and Diederich $M$ : From nature to bedside: Pro-survival and cell death mechanisms as therapeutic targets in cancer treatment. Biotechnol Adv 32: $1111-1122,2014$

23. Natsumeda M, Aoki H, Miyahara H, Yajima N, Uzuka T, Toyoshima Y, Kakita A, Takahashi $\mathrm{H}$ and Fujii Y: Induction of autophagy in temozolomide treated malignant gliomas. Neuropathology 31: 486-493, 2011.

24. Chen X, Wong JY, Wong P and Radany EH: Low-dose valproic acid enhances radiosensitivity of prostate cancer through acetylated p53-dependent modulation of mitochondrial membrane potential and apoptosis. Mol Cancer Res 9: 448-461, 2011.

25. Mari M, Tooze SA and Reggiori F: The puzzling origin of the autophagosomal membrane. F1000 Biol Rep 3: 25, 2011.

26. Levine B and Kroemer G: Autophagy in aging, disease and death: The true identity of a cell death impostor. Cell Death Differ 16: 1-2, 2009

27. Talaber G, Miklossy G, Oaks Z, Liu Y, Tooze SA, Chudakov DM, Banki K and Perl A: HRES-1/Rab4 promotes the formation of LC3(+) autophagosomes and the accumulation of mitochondria during autophagy. PLoS One 9: e84392, 2014.

28. Lai SC and Devenish RJ: LC3-associated phagocytosis (LAP): Connections with host autophagy. Cells 1: 396-408, 2012

29. Palmisano NJ and Meléndez A: Detection of autophagy in Caenorhabditis elegans by western blotting analysis of LGG-1. Cold Spring Harb Protoc 2016: pdb.prot086512, 2016.

30. Klionsky DJ, Abdalla FC, Abeliovich H, Abraham RT, Acevedo-Arozena A, Adeli K, Agholme L, Agnello M, Agostinis P, Aguirre-Ghiso JA, et al: Guidelines for the use and interpretation of assays for monitoring autophagy. Autophagy 8: 445-544, 2012.

31. Lv ZQ, Han JJ, Liu YQ, Wang LL, Tang QL, Sun Q and Li HG: Expression of beclin 1 in non-small cell lung cancer: An immunohistochemical study. Clin Respir J 9: 359-365, 2015.

32. Liang C, Feng P, Ku B, Dotan I, Canaani D, Oh BH and Jung JU: Autophagic and tumour suppressor activity of a novel Beclin1-binding protein UVRAG. Nat Cell Biol 8: 688-699, 2006.

33. Li L, Chen X and Gu H: The signaling involving in autophagy machinery in keratinocytes and therapeutic approaches for skin diseases. Oncotarget: May 12, 2016 (Epub ahead of print).

34. Taneike M, Nishida K, Omiya S, Zarrinpashneh E, Misaka T, Kitazume-Taneike R, Austin R, Takaoka M, Yamaguchi O, Gambello MJ, et al: mTOR hyperactivation by ablation of tuberous sclerosis complex 2 in the mouse heart induces cardiac dysfunction with the increased number of small mitochondria mediated through the down-regulation of autophagy. PLoS One 11: e0152628, 2016.

35. Riquelme I, Tapia O, Espinoza JA, Leal P, Buchegger K, Sandoval A, Bizama C, Araya JC, Peek RM and Roa JC: The gene expression status of the PI3K/AKT/mTOR pathway in Gastric Cancer Tissues and cell lines. Pathol Oncol Res: May 7, 2016 (Epub ahead of print).

36. Feng Y, Backues SK, Baba M, Heo JM, Harper JW and Klionsky DJ: Phosphorylation of Atg9 regulates movement to the phagophore assembly site and the rate of autophagosome formation. Autophagy 12: 648-658, 2016.

37. Malfitano AM, Laezza C, Galgani M, Matarese G, D'Alessandro A, Gazzerro P and Bifulco M: The CB1 receptor antagonist rimonabant controls cell viability and ascitic tumour growth in mice. Pharmacol Res 65: 365-371, 2012. 